\title{
Measurement of the quality of hand contractions
}

\author{
B. W. Duym
}

Department of Electrical Engineering, Bio-information Group. Twente University of Technology. Enschede The Netherlands

\section{G. Pfurtscheller*}

Department of Computing. Institute of Biomedical Engineering. Technical University of Graz, Inffeldgasse 18 A.8010 Graz, Austria

\begin{abstract}
An instrument has been developed which provides quantitative information about hand contractions. The subject must press a rubber balloon for a certain number of times. The information concerning the hand contractions is expressed in terms of certain parameters of the mean pressureftime function. which is generated by the hand contractions of the rubber balloon. The parameters are pressure duration, maximum pressure, pressure integral, pressure increase and pressure decrease. The design is based on a hybrid pressure transducer and a microcomputer. The microcomputer controls the course of the measurement and calculates the paramaters at the end. The small size, only four command switches and the six-digit LED display for parameter presentation make the instrument easy for use in the clinic. Experiments made with the instrument (Elektro. Dynamo-Meter) gave significant measurable differences between the parameters of the dominant and the nondominant hand. A comparative study with previous measurements showed slightly more accurate results with the Elektro-Dynamo-Meter.
\end{abstract}

Keywords - Hand contraction. Microcomputer, Pressure transducer

Med \& Biol Eng \& Comput, 1984, 22, 245-250

\section{Introduction}

PRIOR to voluntary self-paced movements one can observe changes in the central region activity of the human EEG. DEECKE et al. (1976) described an experiment with unipolar EEG measurements on persons performing voluntary rapid finger flexions. By means of a time-reversed analysis of the tape-stored EEG signals slow changes (Bereitschaftspotential, BP) were detected in the DC potential of the EEG signal preceding the rapid finger movement. Other workers (Pfurtscheller and Aranibar, $1980 a ; b$; Pribyl, 1981) investigated the $\mathrm{BP}$ and the change in rhythmic EEG activity (event-related desynchronisation, ERD) in relation to voluntary movements. Bipolar derivations were used in these experiments to determine the topographical distribution of these movement-related phenomena. PRIBYL (1981) reported on the quality of voluntary hand contraction (force, duration, velocity) in relation to the BP and ERD. Data collection and processing for these experiments were carried out by a PDP11/45 computer. To introduce this type of measurement into the clinic a universal hand-contraction meter was developed. The instrument should be easy to use and easy to transport. Direct presentation of certain parameters reflecting information on the force, duration and velocity of the hand movement should be available. This paper describes the functioning of such an instrument, the Elektro-Dynamo-Meter, and reports the results of an investigation with the instrument among 40 healthy persons.

*Correspondence and requests for reprints should be addressed to Dr. Pfurtscheller.

First received 9th March and in final form 9th August 1983.

(C) IFMBE: 1984

\section{Test method}

The hand-movement parameters are derived from a certain number of hand contractions. The subject has to press a rubber balloon for a certain number of times. The balloon must be enclosed by the whole hand, and the contraction must be performed with the whole hand. This method of holding and pressing the balloon appeared to be easily reproducible. The pressure as a function of time (pressure curve, PC), which is developed in the balloon when it is pressed, is recorded by a microcomputer. After recording enough pressure curves, the microcomputer calculates the parameters from the mean pressure curve. Computation of the mean curve from the individual curves requires a certain reproducible event in each pressure curve, which is capable of triggering (starting and stopping) the recording process of the computer. Fig. 1 shows that the trigger events chosen are those moments when the developed pressure rises above, or falls below, a certain threshold (trigger level, TRIGL).

The parameters describing the mean pressure curve are the following:

(a) trigger threshold, TRIGL, in kilopascals $(\mathrm{kPa})$. The threshold is variable and should be adjusted at a minimum level, so that it just does not cause triggering of the computer when the person holds the balloon quietly in the hand

(b) number of recorded pressure curves NPC.

(c) pressure duration, $t_{b a l}(\mathrm{~s})$. As Fig. 1 shows, $t_{b a l}$ is the time interval during which the mean pressure is greater than or equal to TRIGL $\left(t_{1}-t_{0}\right)$.

(d) maximum pressure $X_{\max }(\mathrm{kPa})$ of the mean pressure curve. 
(e) pressure/time area INT (kPas) from $t_{0}$ until $t_{1}$.

( $f$ ) rise, or positive slope, PSL $\left(\mathrm{kPas}^{-1}\right)$ of the mean pressure curve between 25 and 75 per cent of $X_{\max }$.

(g) fall, or negative slope, NSL $\left(\mathrm{kPas}^{-1}\right)$ of the mean pressure curve between 25 and 75 per cent of $X_{\max }$.

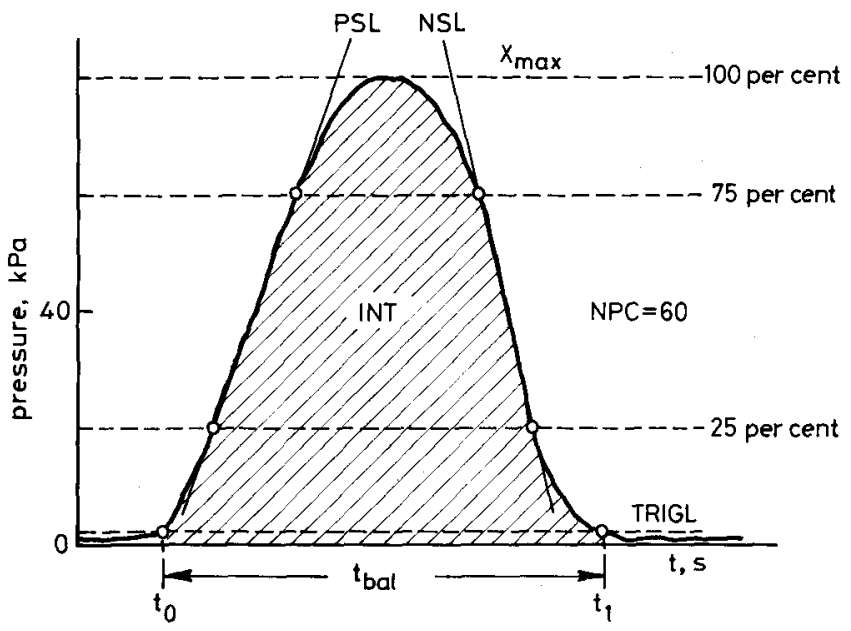

Fig. 1 Typical mean pressure curve from 60 hand contractions of a healthy person. The symbols are the parameters used

The first two parameters serve to control the measurement. They give the possibility of comparing the parameters of different persons or of the same person after a lapse of time (interpersonal or intrapersonal comparison). The last five parameters are suitable for quantification of the hand contraction.

Computation of the parameters from the mean pressure curve implies a certain sensitivity of the parameters $X_{\max }$, PSL and NSL to nonuniformity (time variations) between the single pressure curves. If two curves have the same maxima which do not occur at exactly the same time in relation to the starting point $t_{0}$, the mean pressure curve will always have a smaller maximum value than the mean maximum of both pressure curves. This effect becomes stronger for events further away from $t_{0}$, such as NSL. The parameter NSL is then more of a measure for uniformity between the single pressure curves than for the mean velocity of the hand contractions. Another method, which is not sensitive to nonuniformity (PRIBYL, 1981), is the calculation of the mean parameters from the parameters of each single pressure curve. However, this method is less accurate for the slope calculations of the very weak hand contractions developed by patients.

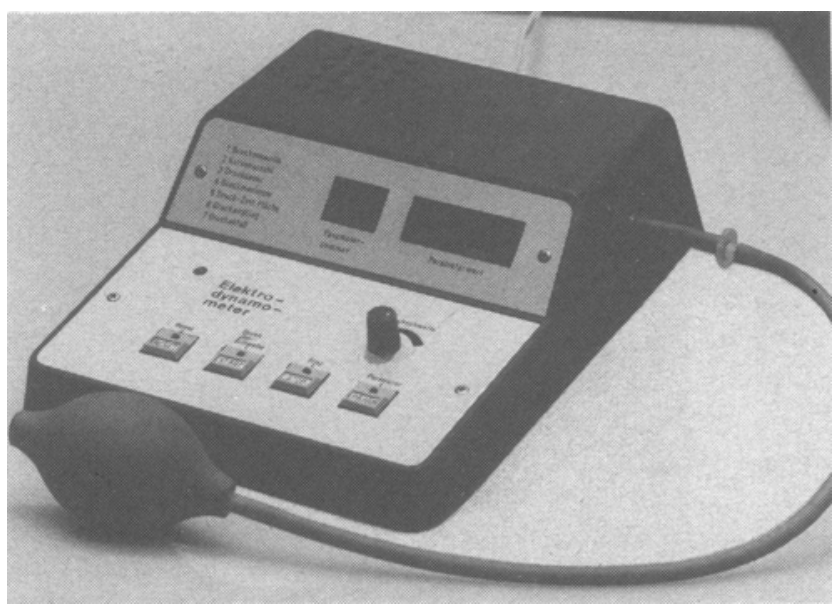

Fig. 2 The Elektro-Dynamo-Meter

\section{Test procedure}

The procedure starts in the RESET mode with adjustment (by the operator) of the threshold TRIGL. The adjusted value is shown on the display. An indicator LED on the control panel indicates whether the applied pressure is above the threshold. If this occurs in the RESET mode, the applied pressure appears on the display. This gives an initial impression of the pressure which can be developed by the subject.

The actual pressure curve recording proceeds in the START mode. The display shows the number of recorded pressure curves NPC, which has an initial value of zero. After $3 \mathrm{~s}$ the display is turned off. This signals to the subject that the balloon can now be pressed. After recording the pressure curve, the display lights up again for $3 \mathrm{~s}$, indicating the new NPC.

During these $3 \mathrm{~s}$ the recording of a pressure signal is inhibited. This forces the subject to take a minimum pause

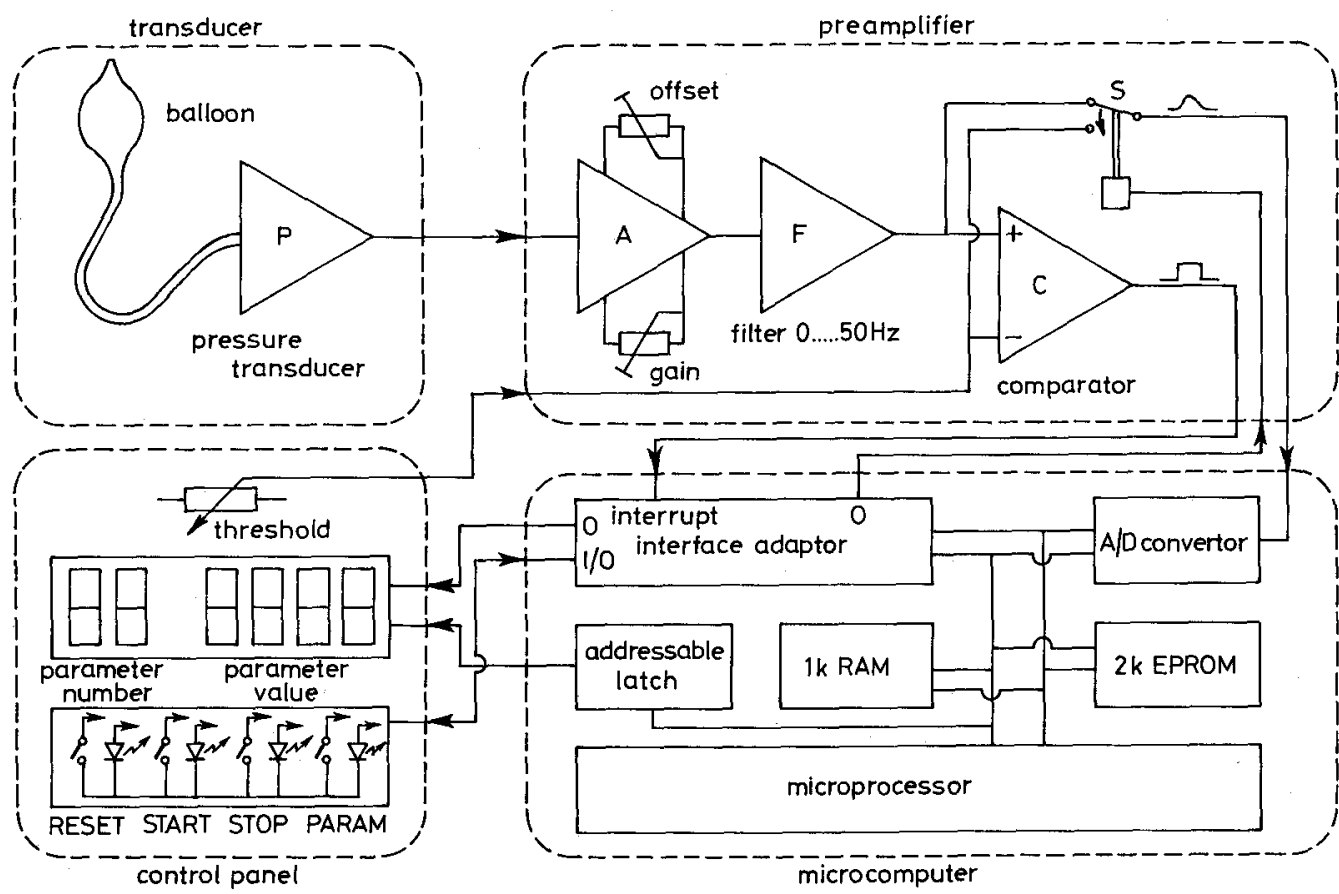

Fig. 3 Block diagram of the ElektroDynamo-Meter 
(longer is always possible) between two hand contractions. The instrument is shown in Fig. 2.

This recording sequence can be repeated until enough pressure curves are recorded. In the STOP mode the computer calculates the parameters. Presentation of the parameter values on the display occurs in a numbered order (see Section 2) by repeatedly pressing the PARAM button. One entire parameter test for both hands, using the maximum of 64 pressure curves, including instruction of the subject, takes about $10 \mathrm{~min}$.

\section{Design}

The design of the Elektro-Dynamo-Meter can be subdivided into four different blocks. Fig. 3 shows the transducer part, the preamplifier, the microcomputer and the control panel. The control panel consists of the four command switches with indicator LEDs, the six-digit display and the TRIGL adjustment.

The balloon/tube transducer system is filled with air. The internal and external dimensions of the system appeared to have a substantial influence on the parameter values. This is not too serious when the same balloon and tube are always used, since the measurements are always relative to previous measurements. Nevertheless, there are still some practical conditions to be met for the balloon/tube transducer system. First, the balloon must be large enough that it always fills the whole hand, even when maximum force is applied. Thus the external volume of the balloon must be large. The maximum pressure is then limited by the hand force of the subject, and not by the fact that the balloon is too small to be compressed further. Secondly, the internal volume of the balloon/tube transducer system must be small enough that a small contraction of the hand leads to a relatively high change of the internal volume of (and thus a high change of the pressure in) the system. Maximum pressure is then obtained from a small hand contraction, which limits the deformation of the balloon.

The balloon and tube used for the Elektro-DynamoMeter satisfy both conditions. They are normally used as part of a blood-pressure meter. The balloon contains a valve which ensures equality of the internal and external pressures when the balloon is not pressed, and thus the same shape of the balloon under all ambient pressure circumstances. The valve closes at a pressure difference approximately equal to $0.5 \mathrm{kPa}$, which is lower than the minimum threshold (TRIGL), which can be adjusted. This and the fact that the pressure transducer measures a pressure difference relative to the ambient pressure allows the measurements with the Elektro-Dynamo-Meter to be independent of the ambient pressure.

The hybrid pressure transducer $P$ (National Semiconductor LX $1603 \mathrm{~GB}$ ) has an offset voltage which is rather sensitive to temperature. Heat generation in the instrument causes a change of the offset level of approximately $2 \mathrm{kPa}$ within half an hour, which is more than 1.5 per cent of the pressure range $(0 \ldots 130 \mathrm{kPa})$. This temperature drift is continuously corrected by the micro-computer. Whenever the balloon is not pressed the micro-computer measures the pressure signal and takes that value as the zero pressure level.

After offset adjustment, amplification (A) and filtering (F), the pressure signal is compared $(C)$ with the threshold TRIGL. The trigger pulse derived by the comparator starts (positive edge) and stops (negative edge) the pressure curve recording via an interrupt input of the interface adapter of the microcomputer. The analogue-to-digital convertor is connected $(\mathrm{S})$ either to the pressure signal for pressure measurement or to the threshold signal for measurement of the trigger threshold.

The microcomputer is a general-purpose design from the Technical University of Graz (WIEsSPEINER et al., 1979), equipped with a 6502 processor, 6522 versatile interface adapter (Mostek), RAM, EPROM, analogue-to-digital convertor and addressable latch. Optimal use of the available RAM allows recording of the pressure curves with a simple frequency of $128 \mathrm{~Hz}$ (maximum duration of $2 \mathrm{~s}$ per pressure curve). This ensures an optimal reproducibility for parameter measurements of very fast hand contractions (minimum duration of $0.2 \mathrm{~s}$ ). If necessary the microcomputer can easily be extended with 1 kbyte RAM, 2 kbyte EPROM, an interrupt controller and two digital-to-analogue convertors. Because the functional performance of the ElektroDynamo-Meter is determined by the application of the microcomputer, the use of other software (EPROM) and/or other components allows flexible adaptation according to the wishes of the user, as, for example, output presentation on a printer or plotter, or calculation of parameters other than those mentioned in Section 2.

\section{Software program}

The test procedure is determined by the software program. Fig. 4 shows the flow diagram of the main program, which is the test procedure described in Section 3. This program is started when the RESET button is pressed.

The trigger pulse derived by the comparator ( $\mathrm{C}$ in Fig. 3 ) indicates that a pressure curve is being generated. The positive edge (from low to high) of the pulse marks the beginning of a pressure curve, and the negative edge (from high to low) marks the end. The pulse is led to one of the interrupt inputs of the interface adaptor. The interrupt input

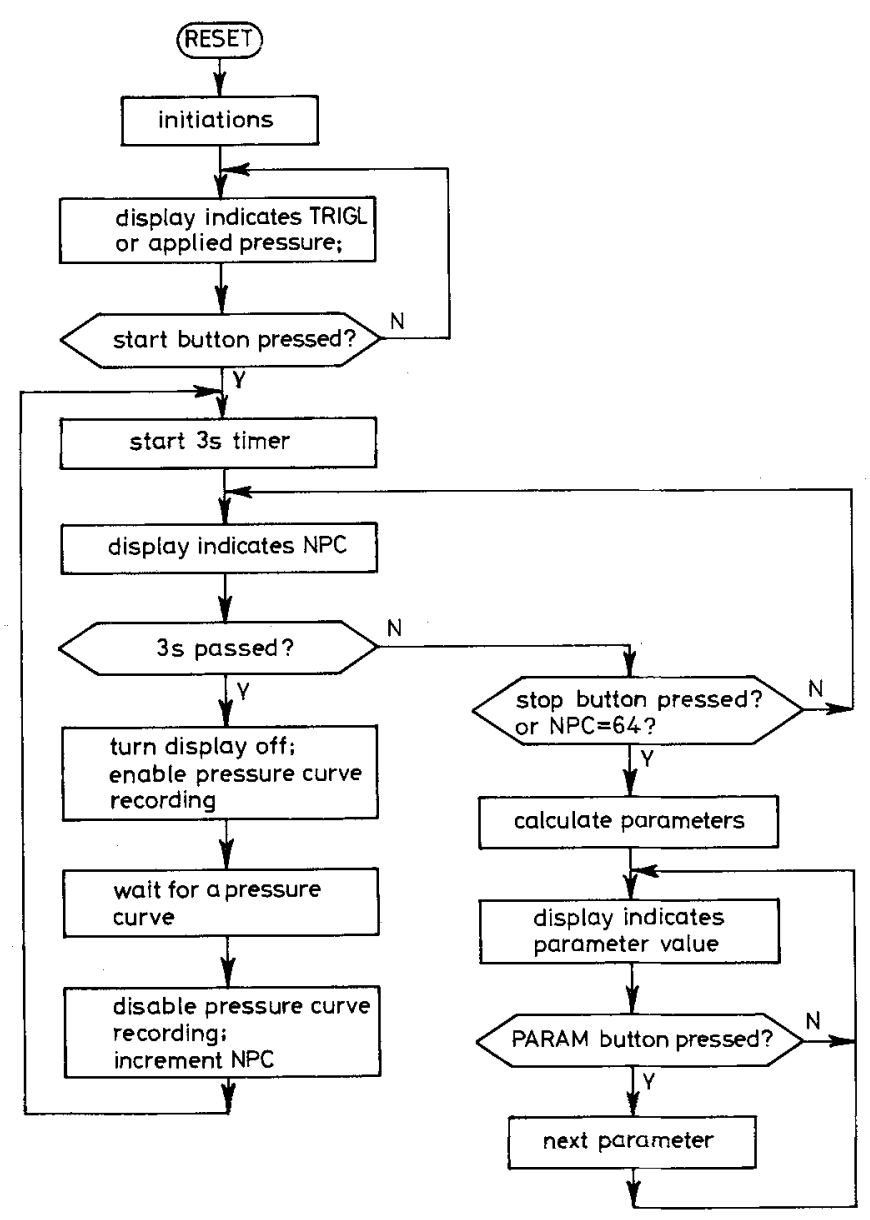

Fig. 4 Flow diagram of the main program 
is sensitive either to a positive edge, or to a negative edge. Selection is under software control. The initiation after pressing the RESET button sets the sensitivity on the positive edge. The interrupt service routine, which is activated when the selected edge form occurs (see Fig. 5), starts with checking whether the sensitivity is set on a positive edge. If that is true, it means the beginning of a pressure curve. The routine initiates and starts the $128 \mathrm{~Hz}$ sample timer, and changes the sensitivity to the negative edge. If the sensitivity was set on a negative edge, it means the end of a pressure curve. In this case, the routine stops the sample timer and changes the sensitivity to the positive edge. During this phase of the test procedure, the main program (Fig. 4) remains in the waiting loop 'wait for the pressure curve'. The inhibition of the pressure curve recording ("disable pressure curve recording' in Fig. 4) during the pause of $3 \mathrm{~s}$ between two hand contractions is obtained by masking the recognition of an interrupt signal.

The $128 \mathrm{~Hz}$ sample timer is an interval timer operating on an interrupt basis, in the repeated mode. Once the timer is enabled, it generates 128 interrupt signals per second, until the timer is disabled. The timer interrupt service routine takes a sample from the pressure curve, and stores it in the pressure curve array. This array contains 256 elements. When the last element is filled and the pressure curve is not yet ended, the routine disables the sample timer. This means that a maximum recording time of $2 \mathrm{~s}$ is available for the pressure curves of one test to be added together in the pressure curve array. Thus, at the end of a test, the array contains the sum pressure curve. The parameters are then calculated from this sum pressure curve.

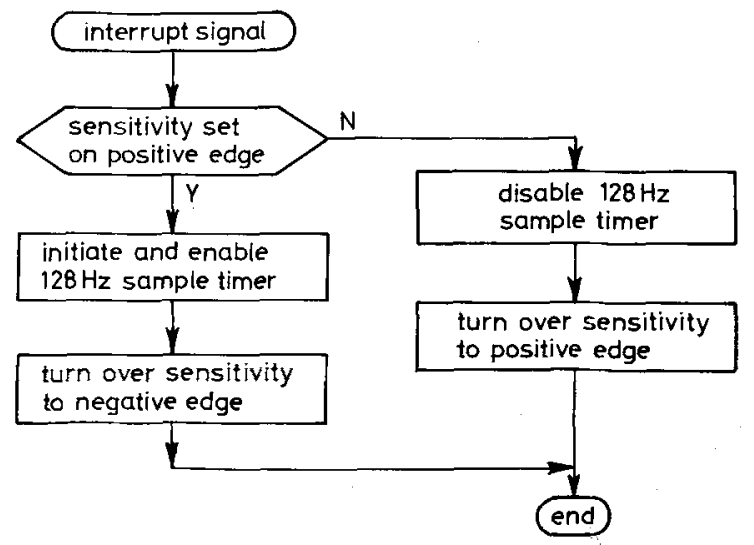

Fig. 5 Flow diagram of the interrupt service routine, which starts and stops the recording of a pressure curve

Table 1 Results of 20 tests with three different balloons

\begin{tabular}{|c|c|c|c|c|c|c|}
\hline & \multicolumn{2}{|c|}{ Balloon 1} & \multicolumn{2}{|c|}{ Balloon 2} & \multicolumn{2}{|c|}{ Balloon 3} \\
\hline & \multicolumn{2}{|c|}{$\begin{array}{l}O V=33 \\
I V=20\end{array}$} & \multicolumn{2}{|c|}{$\begin{array}{l}\mathrm{OV}=65 \\
\mathrm{IV}=33\end{array}$} & \multicolumn{2}{|c|}{$\begin{aligned} \mathrm{OV} & =113 \\
\mathrm{IV} & =92\end{aligned}$} \\
\hline & $\begin{array}{c}X_{\max } \\
(\mathrm{kPa})\end{array}$ & $\begin{array}{c}\text { INT } \\
(\mathrm{kPas})\end{array}$ & $\begin{array}{c}X_{\max } \\
(\mathrm{kPa})\end{array}$ & $\begin{array}{c}\text { INT } \\
(\mathrm{kPas})\end{array}$ & $\begin{array}{c}X_{\max } \\
(\mathrm{kPa})\end{array}$ & $\begin{array}{c}\text { INT } \\
(\mathrm{kPa} \mathrm{s})\end{array}$ \\
\hline$X$ & $82 \cdot 2$ & 155 & 96.5 & 184 & $86 \cdot 8$ & 161 \\
\hline SD & 5.67 & $12 \cdot 4$ & 2.65 & $8 \cdot 4$ & $4 \cdot 40$ & $9 \cdot 6$ \\
\hline $\mathrm{SD}, \%$ & $6 \cdot 9$ & $7 \cdot 9$ & $2 \cdot 7$ & $4 \cdot 6$ & $5 \cdot 1$ & $6 \cdot 0$ \\
\hline
\end{tabular}

IV tube $=10 \mathrm{~cm}^{3}$

TRIGL $=3 \mathrm{kPa}$

$\mathrm{NPC}=10$

persons: 1

age: 26

$\mathrm{OV}=$ outer volume in $\mathrm{cm}^{3}$

IV = inner volume in $\mathrm{cm}^{3}$

\section{Results}

In Section 4 we mentioned the effect of different types of balloon and tube on the parameter values. The effect of the size of the balloon was investigated in an experiment with three different balloons with the same tube. Twenty tests were made with each balloon by the same person, over a period of three weeks. Since our interest was mainly focused on the transformation of hand force into pressure, only the paramaters $X_{\max }$ and INT were taken into account. One test consisted of ten hand contractions $(\mathrm{NPC}=10)$, each as powerful as possible and for a duration of at least $2 \mathrm{~s}$. Timing of the $2 \mathrm{~s}$ recording period was controlled by the microcomputer. This eliminated time as a variable in the parameter INT.

Table 1 lists the mean parameter value $(\mathrm{X})$, standard deviation (SD) and the standard deviation as percentage of the mean (SD, per cent), over the 20 tests for each balloon. Most important is the lower value of the (interpersonal) standard deviation, which is obtained with balloon 2. This points to the ability of a more accurate parameter estimation with this balloon. The external volume of this balloon is large enough to fill the whole hand, and the internal volume is small enough that only a small hand contraction is necessary to reach a high pressure change. Balloon 1, which was used for previous measurements (PRIBYL, 1981), is too small. It does not fill the whole hand, particularly not when maximum force is applied. Therefore the results for $X_{\max }$ and INT are lower than those obtained with balloon 2 . The internal volume of balloon 3 is too large, so that large hand contractions are necessary to reach a certain pressure in the system. For these reasons, balloon 2 was used in the construction of the Elektro-Dynamo-Meter.

Reference data about hand contractions for subsequent clinical studies were obtained in an experiment with 40 healthy volunteers. The persons were asked to press the balloon 30 times $(\mathrm{NPC}=30$ ) with each hand. The hand contractions should be powerful and fast and should be kept as uniform as possible. Before every parameter test the persons were asked which hand they thought would be their dominant hand. This gave the results for the dominant (D) and nondominant (ND) hands listed in Table 2 .

Except for the parameter $T_{b a l}$, all parameters showed a

Table 2 Mean value $(X)$, standard deviation $(S D)$ and standard deviation as a percentage of the mean ( $S D$, per cent) for an investigation with 40 healthy persons for the dominant $(D)$ and nondominant hand (ND)

\begin{tabular}{|c|c|c|c|c|c|c|}
\hline & & $\begin{array}{l}T_{b a l} \\
(\mathrm{~s})\end{array}$ & $\begin{array}{c}X_{\max } \\
(\mathrm{kPa})\end{array}$ & $\begin{array}{c}\text { INT } \\
(\mathrm{kPas})\end{array}$ & $\begin{array}{c}\text { PSL } \\
\left(\mathrm{kPa} \mathrm{s}^{-1}\right)\end{array}$ & $\begin{array}{c}\mathrm{NSL} \\
\left(\mathrm{kPa} \mathrm{s}^{-1}\right)\end{array}$ \\
\hline \multirow{3}{*}{ D } & $X$ & 0.54 & $87 \cdot 0$ & $29 \cdot 7$ & 602 & 608 \\
\hline & SD & 0.23 & $20 \cdot 6$ & $15 \cdot 1$ & 192 & 264 \\
\hline & $\mathrm{SD}, \%$ & 42 & 24 & 51 & 32 & 43 \\
\hline & $\mathrm{X}$ & 0.54 & 76.9 & $26 \cdot 5$ & 534 & 497 \\
\hline \multirow{2}{*}{ ND } & $\mathrm{SD}$ & $0 \cdot 21$ & $18 \cdot 0$ & $12 \cdot 1$ & 173 & 231 \\
\hline & $\mathrm{SD}, \%$ & 39 & 24 & 46 & 32 & 47 \\
\hline
\end{tabular}

TRIGL $=3 \mathrm{kPa}$

$\mathrm{NPC}=30$

persons: 40

male: 25

female: 15

age: $28 \pm 7$

right handed: 33

left handed: 4

double handed: 3 
highly significant difference (Wilcoxon test, $p=0.01$ per cent) between the dominant and nondominant hands.

Nine persons in the group said that they were double handed or that they practised a lot with both hands because of their profession or hobbies (typists, musicians). Special study of the results of these persons (Table 3 ) showed no significant differences between both hands.

Table 3 Mean value $(X)$, standard deviation (SD) and standard deviation as a percentage of the mean ( $S D$, per cent) for parameter tests with specially selected (double handed, typists, musicians) healthy persons for the dominant $(D)$ and nondominant hand $(N D)$

\begin{tabular}{|c|c|c|c|c|c|c|}
\hline & & $\begin{array}{l}t_{b a l} \\
(\mathrm{~s})\end{array}$ & $\begin{array}{r}X_{\max } \\
(\mathrm{kPa})\end{array}$ & $\begin{array}{c}\text { INT } \\
(\mathrm{kPas})\end{array}$ & $\begin{array}{c}\text { PSL } \\
\left(\mathrm{kPa} \mathrm{s}^{-1}\right)\end{array}$ & $\begin{array}{c}\mathrm{NSL} \\
\left(\mathrm{kPa} \mathrm{s}^{-1}\right)\end{array}$ \\
\hline \multirow{3}{*}{ D } & $X$ & 0.49 & $80 \cdot 3$ & $24 \cdot 9$ & 609 & 597 \\
\hline & $\mathrm{SD}$ & $0 \cdot 18$ & $15 \cdot 2$ & $8 \cdot 5$ & 183 & 262 \\
\hline & $\mathrm{SD}, \%$ & 37 & 19 & 34 & 30 & 44 \\
\hline \multirow{3}{*}{ ND } & X & 0.46 & 78.0 & $23 \cdot 1$ & 594 & 611 \\
\hline & $\mathrm{SD}$ & 0.16 & $12 \cdot 8$ & $6 \cdot 6$ & 188 & 289 \\
\hline & $\mathrm{SD}, \%$ & 36 & 16 & 29 & 32 & 47 \\
\hline
\end{tabular}

TRIGL $=3 \mathrm{kPa}$

$\mathrm{NPC}=30$

persons: 9

male: 5

female: 4

age: $27 \pm 6$

right handed: 5

double handed: 3

left handed: 1

Besides the fact that the results in Table 2 showed a lower force and velocity for the nondominant hand, the higher value of the difference PSL-NSL for the nondominant hand $(p=1$ per cent), pointed to less uniformity in the hand contractions performed with this hand. Again, this difference between both hands was not significant in the group 'doublehanded persons'.

The reproducibility of the parameters in the test described for Table 2 was studied in the third experiment. Eight persons made ten tests with the dominant hand in a period of one week. The intrapersonal standard deviations are shown in Table 4.

We see that all standard deviations for all parameters are much smaller (3-10 times) than the interpersonal standard deviation in Table 2, which means that the parameters can be called characteristic for a person. The parameters $X_{\max }$ and PSL in particular can be estimated very accurately with this type of test. It was remarkable in this experiment that all persons had a slightly positive trend in the NSL value, whereas the other parameters did not show any tendency. Because of the many tests over a short period, persons 'learned' to perform hand contractions with a higher degree of uniformity. This explains the relatively high standard deviations for the NSL parameters.

\section{Discussion}

The measurements made with the Elektro-DynamoMeter confirmed the expected lower values for force and velocity of the nondominant hand in comparison with the dominant hand of healthy persons. These results agree with earlier findings (PRIBYL, 1981) and are even more significant ( $p=0.01$ per cent against $p=1-5$ per cent). The more accurate results with the Elektro-Dynamo-Meter are primarily caused by the use of another balloon. Apart from the subjective experience that the new balloon (balloon 2 instead of balloon 1 in Table 1) 'feels more comfortable in the hand', it also proved to give a better reproducibility and thus a better estimation of the parameters. The choice of another balloon, however, reduces the ability to compare our results with the results of PrIBYL (1981), but this does not outweight the gain in accuracy obtained with the new balloon. Moreover, the comparison of the results of both investigations was already hindered by the use of other units. Pribyl (1981) used $2 \mathrm{kp} \mathrm{cm}^{-2}$ as the pressure unit whereas the Elektro-Dynamo-Meter uses the SI unit (kilo) pascal $\left(1 \mathrm{kpcm}^{-2}=98.07 \mathrm{kPa}\right)$.

Table 4 Intrapersonal standard deviations as percentage of the mean parameter values over ten tests for eight healthy persons with the dominant hand

\begin{tabular}{|c|c|c|c|c|c|}
\hline Person & $\begin{array}{c}t_{b a l} \\
(\text { per cent) }\end{array}$ & $\begin{array}{c}X_{\max } \\
\text { (per cent) }\end{array}$ & $\begin{array}{c}\text { INT } \\
\text { (per cent) }\end{array}$ & $\begin{array}{c}\text { PSL } \\
\text { (per cent) }\end{array}$ & $\begin{array}{c}\text { NSL } \\
\text { (per cent) }\end{array}$ \\
\hline 1 & 8.7 & $3 \cdot 0$ & $10 \cdot 2$ & 3.9 & $15 \cdot 8$ \\
\hline 2 & $9 \cdot 1$ & $3 \cdot 2$ & $12 \cdot 1$ & $3 \cdot 5$ & 11.5 \\
\hline 3 & $10 \cdot 0$ & $4 \cdot 1$ & 16.4 & $3 \cdot 2$ & $8 \cdot 3$ \\
\hline 4 & 9.8 & $2 \cdot 9$ & $13 \cdot 2$ & $3 \cdot 3$ & $15 \cdot 2$ \\
\hline 5 & $10 \cdot 7$ & $2 \cdot 9$ & 14.4 & 4.0 & $10 \cdot 2$ \\
\hline 6 & $10 \cdot 6$ & $4 \cdot 0$ & $15 \cdot 3$ & 3.8 & 11.8 \\
\hline $7^{*}$ & $7 \cdot 2$ & $2 \cdot 5$ & $9 \cdot 1$ & $2 \cdot 4$ & $9 \cdot 4$ \\
\hline 8 & 8.0 & $4 \cdot 2$ & 9.7 & $4 \cdot 0$ & $10 \cdot 6$ \\
\hline
\end{tabular}

TRIGL $=3 \mathrm{kPa}$

$\mathrm{NPC}=30$

persons: 8

male: 5

female: 3

right handed: 6

left handed: 2

age: $27 \pm 9$

$*=$ person of Table 1

Another difference between both investigations is the method of averaging for the parameter calculations (see Section 2). Although we do not have proof, because we could not involve patients in our experiments, we expect better results with the averaging method we used for the ElektroDynamo-Meter. Our method, however, leads to another interpretation of the parameter NSL. In PRIBYL (1981), NSL and PSL stand for the mean velocity of the hand contractions. NSL is always larger (1.25-1.5 times) than PSL. With our method of averaging, NSL is more a measure of the uniformity of the hand contractions. Less uniformity causes a strong decrease of NSL, and to a lesser degree also of $X_{\max }$ and PSL (not for INT). The results for PSL and NSL in Table 2 therefore point to less uniformity in the hand contractions of the nondominant hand. In summary, we can say that differences in the parameters $X_{\max }$, PSL and NSL between the dominant and nondominant hand can be caused by differences in force, velocity and uniformity. The fact that the parameter INT, which is independent of uniformity, has the same proportional difference between both hands as $X_{\max }$ and PSL shows that the differences between both hands for $X_{\max }$ and PSL are due to differences in velocity and force. The difference between both hands for NSL is mainly due to difference in uniformity of the hand contractions.

For the tests summarised in Tables 2-4, subjects were instructed 'to press as powerfully, quickly and uniformly as possible'. Such a test gave accurate results for the parameters $X_{\max }$ and PSL and thus for force and velocity. This test contains a compromise between the two variables. Other kinds of tests in which only the force, or only the velocity or only the duration is important, would give different results 
with different estimation accuracies for the parameters (e.g. INT in Table 1). Therefore a single parameter cannot be seen as an absolute measure, but it may be used to compare interor intrapersonal differences.

\section{References}

Deecke, L., Grozinger, B. and Kornhuber, H. H. (1976) Voluntary finger movement in man: cerebral potentials and theory. Biol. Cybernetics, 23, 99-119.
Pfurtscheller, G. and Aranibar, A. (1980a) Changes in central EEG activity in relation to voluntary movement $\mathbf{I}$. normal subjects. Prog. in Brain Res., 54, 22-231.

Pfurtscheller, G. and Aranibar, A. (1980b) Changes in central EEG activity in relation to voluntary movement II. hemiplegic patients. Ibid., 54, 491-495.

PRIBYL, W. (1981) Lokalisation von Quellen bioelektrischer Hirnaktivitäten unter besonderer Berücksichtigung von Willkürbewegungen. Dissertation TU Granz, 13-98.

Weisspeiner, G., Gruber, H. and SchuY, S. (1979) Modulares hierarchisches Multiprozessorsystem mit gemeinsamen Arbeitsspeicher. Informationstagung Mikroelektronik 1979, Wien, $176-179$. 